\title{
Mastocitose em idade pediátrica: Caracterização de um período de 5 anos num hospital central
}

\section{Mastocytosis during pediatric age: 5-year characterization in a central hospital}

Data de receção / Received in: I I/8/2020

Rev Port Imunoalergologia 2021;29 (2):85-93

Francisca Cunha', Carmelita Ribeiro', Leonor Ramos², Jóni Carvalho', Ana Todo Bom'

I Serviço de Imunoalergologia, Centro Hospitalar e Universitário de Coimbra

2 Serviço de Dermatologia, Hospital Pediátrico, Centro Hospitalar e Universitário de Coimbra

Prémio Leti-SPAIC

\section{RESUMO}

Fundamentos: A mastocitose é um grupo de doenças raras caracterizada pela expansão e acumulação excessiva de mastócitos clonais, de que resulta o envolvimento cutâneo e/ou sistémico. Nas idades pediátricas, a forma mais comum de mastocitose é a mastocitose cutânea. Objetivos: $O$ presente trabalho tem como objetivo avaliar as características demográficas e os achados clínicos em crianças com diagnóstico de mastocitose. Métodos: Foram revistos os processos clínicos, desde janeiro de 2014 a junho de 2019, de todos os doentes com diagnóstico de mastocitose no Hospital Pediátrico de Coimbra. Resultados: Os 14 doentes foram diagnosticados com mastocitose cutânea. A idade média de início das lesões foi de 15, I meses, sendo a mediana de 5 meses. A idade média de diagnóstico foi de 22,5 meses e a mediana de 9,5 meses. Dos 14 doentes, 8 eram do sexo masculino. 12 doentes tinham mastocitose cutânea maculopapular e 2 tinham mastocitoma cutâneo. No que diz respeito à subclassificação das variantes da MC maculopapular, II foram classificadas como polimórficas e I como monomórfica. O sinal de Darier foi positivo em 12 doentes, 10 tinham prurido, 2 apresentavam dor abdominal e 3 diarreia. Não foi constatado nenhum episódio de anafilaxia. Em todos os doentes foi realizado um estudo analítico inicial, a destacar o doseamento da triptase sérica, que variou entre I,3-15,9 ng/mL na primeira 
avaliação. Em 8 doentes, o diagnóstico foi confirmado através de biópsia cutânea. A terapêutica baseou-se na evicção dos fatores desencadeantes, em anti-histamínicos $\mathrm{H}_{1}$ e corticosteroide tópico de potência moderada a forte. $O$ curso das lesões permanece estável em 12 doentes. Conclusões: $O$ presente trabalho corrobora o que está descrito na literatura corrente sobre o tema. Realça a importância do conhecimento das características da doença em idade pediátrica para a prevenção de fatores desencadeantes, diagnóstico, investigação e tratamento dirigidos.

Palavras-chave: Mastocitose, mastocitose cutânea, mastocitose pediátrica.

(c) 202I Sociedade Portuguesa de Alergologia e Imunologia Clínica. Publicado por Publicações Ciência \& Vida. Este é um artigo Open Access sob uma licença CC BY-NC-ND (http://creativecommons.org/licenses/by-nc-nd/4.0/).

\section{ABSTRACT}

Background: Mastocytosis is a group of rare diseases, characterized by the excessive clonal mast cell proliferation and accumulation, having cutaneous and/or systemic involvement. During pediatric age, cutaneous mastocytosis is the most common presentation. Aim: This descriptive analysis of children diagnosed with mastocytosis has the purpose of evaluating their demographic characteristics and clinical findings. Methods: The clinical files of patients diagnosed with mastocytosis from January 2014 to June 2019 at Hospital Pediátrico de Coimbra were reviewed. Results: Cutaneous mastocytosis was the final diagnosis for all 14 patients. The mean age for the starting lesions was 15 .I months, with a median of 5 months. The mean age for diagnosis was 22.5 months and the median 9.5 months. 8 patients were male, 12 had maculopapular cutaneous mastocytosis and 2 had mastocytoma. Regarding the subclassification of maculopapular cutaneous mastocytosis, II were classified as polymorphic and I as monomorphic. Darier's sign was positive in 12 patients, I0 had pruritus, 2 had abdominal pain and 3 diarrhea. None of the patients had an anaphylactic episode. Every patient had an initial evaluation with tryptase dosage measurement, varying between $1.3-15.9 \mathrm{ng} / \mathrm{mL}$ at this point. 8 patients had the diagnosis confirmed through a skin biopsy. As medical therapy was included avoidance of triggers, $\mathrm{HI}$ antihistamine and moderate to strong strength topical corticosteroid. The lesions progression remains stable in 12 patients. Conclusions: This analysis reaffirms what is written about the theme in current literature. It emphasizes the importance of knowing the disease's pediatric characteristics to ensure the prevention of triggers and an accurate diagnosis, investigation and treatment.

Key-words: Cutaneous mastocytosis, mastocytosis, pediatric mastocytosis.

(C) 202I Sociedade Portuguesa de Alergologia e Imunologia Clínica. Published by Publicações Ciência \& Vida. This is an open access article under the CC BY-NC-ND license (http://creativecommons.org/licenses/by-nc-nd/4.0/). 


\section{INTRODUÇÃO}

$E^{2}$ m 1878, Paul Ehrlich descreveu, pela primeira vez, os mastócitos'. Unna, em 1887, relata a presença de numerosos mastócitos em lesões cutâneas de urticária pigmentosa, termo atualmente em desuso. Contudo, o termo mastocitose apenas é descrito, pela primeira vez, em 1936'. A mastocitose representa um grupo de doenças, sendo caracterizada pela sobreativação e/ou expansão e acumulação de mastócitos clonais em diferentes órgãos e sistemas ${ }^{2}$.

A etiologia da mastocitose, na maioria dos doentes, decorre de uma mutação ativadora do ckit, com função de cinase de tirosina transmembranar, sendo que na presença de algumas mutações existe um aumento da sensibilidade do stem cell factor (SCF). A ligação do SCF-ckit, em condições fisiológicas, promove o crescimento, proliferação, diferenciação, sobrevivência dos mastócitos e a libertação de mediadores mastocitários. No adulto, a mutação mais comum resulta da substituição da valina pelo aspartato no codão 816 do KIT (D8I6V) ${ }^{3}$. Na população pediátrica, a mutação KIT D8I6V é detetada aproximadamente em $25 \%$ a $35 \%$ das crianças com mastocitose, sendo que as mutações nos exões 8 , 9 e II do KIT são mais prevalentes na população pediátrica, em comparação com os adultos ${ }^{4}$.

No que respeita às manifestações clínicas, a mastocitose pode apresentar-se com máculas e pápulas, ou mesmo nódulos, de tonalidade eritemato-acastanhada e superfície "semelhante a couro", com eritema e urticariação espontâneas ou após estímulo, bem como náuseas, vómitos, diarreia e/ou dor abdominal, entre outros. Pode atingir vários órgãos e sistemas, como pele, medula óssea, trato gastrointestinal, sistema cardiovascular, sistema nervoso central e sistema músculo-esquelético ${ }^{5}$.

A mastocitose pode ser classificada em diversas variantes de acordo com a apresentação clínica, achados patológicos e prognóstico. De acordo com a classificação da Organização Mundial de Saúde (OMS) de 2016, a mastocitose divide-se em mastocitose cutânea
(MC), quando há apenas envolvimento cutâneo, mastocitose sistémica (MS), quando há envolvimento extracutâneo com ou sem envolvimento cutâneo concomitante, e sarcoma mastocitário². A mastocitose cutânea divide-se em maculopapular, mastocitoma cutâneo e mastocitose cutânea difusa. A mastocitose sistémica divide-se em indolente, quiescente, agressiva, mastocitose sistémica com neoplasia hematológica associada e leucemia mastocitária (Quadro I)². O diagnóstico de mastocitose cutânea é estabelecido pela presença de lesões cutâneas características (critério major), associadas a pelo menos um critério minor (Quadro 2$)^{6}$. São critérios de diagnóstico de mastocitose sistémica a presença do critério major associado a um critério minor ou a presença de pelo menos três critérios minor (Quadro 2) ${ }^{2,7}$.

A prevalência da mastocitose em idade pediátrica não é clara; estima-se que a prevalência na idade adulta seja de 10 casos por 100000 habitantes e acredita-se que a prevalência na população pediátrica seja semeIhante ou superior ${ }^{8}$. A mastocitose cutânea é frequentemente diagnosticada na infância, sendo que a maioria das crianças apresenta lesões cutâneas nos 2 primeiros anos de vida e 10 a $20 \%$ das lesões cutâneas estão presentes ao nascimento'. $\mathrm{Na}$ maioria dos casos, as lesões desaparecem durante a puberdade?. Uma vez que se trata de uma doença com possível progressão

Quadro I. Classificação de mastocitose de acordo com a classificação da OMS (2016) ${ }^{2}$

\section{Mastocitose cutânea (MC) \\ - MC maculopapular \\ - MC difusa \\ - Mastocitoma cutâneo}

2. Mastocitose sistémica (MS)

- MS indolente

- MS quiescente

- MS agressiva

- MS com neoplasia hematológica associada

- Leucemia mastocitária

3. Sarcoma mastocitário 
Quadro 2. Critérios de diagnóstico de mastocitose cutânea e sistémica ${ }^{2,9}$

\begin{tabular}{|c|c|}
\hline \multicolumn{2}{|r|}{$\begin{array}{l}\text { I. Mastocitose cutânea (MC) } \\
\text { Ausência de critérios para estabelecer o diagnóstico de MS }\end{array}$} \\
\hline Critério major & Presença de lesões cutâneas características de MC. \\
\hline Critérios minor & $\begin{array}{l}\text { a. Achado histológico de um infiltrado monomórfico de mastócitos consistindo em grandes agregados } \\
\text { de mastócitos positivos para triptase ( }>15 \text { células/agregado) ou }>20 \text { mastócitos dispersos por campo } \\
\text { microscópico de alta resolução. } \\
\text { b. Deteção, na lesão cutânea, da mutação no codão } 816 \text { do gene KIT. }\end{array}$ \\
\hline \multicolumn{2}{|r|}{ 2. Mastocitose sistémica (MS) } \\
\hline Critério major & $\begin{array}{l}\text { Infiltrados mastocitários multifocais ( } \geq 15 \text { mastócitos nos agregados) na biópsia de medula óssea e/ou } \\
\text { noutros órgãos extracutâneos. }\end{array}$ \\
\hline Critérios minor & $\begin{array}{l}\text { a. > de } 25 \% \text { dos mastócitos são atípicos no aspirado de medula óssea ou em forma de fuso em infiltrados } \\
\text { detetados em órgãos viscerais. } \\
\text { b. Deteção da mutação ativadora no codão } 816 \text { do KIT na medula óssea ou noutro órgão extracutâneo. } \\
\text { c. Expressão de CD2 e/ou CD25 nos mastócitos na medula óssea, sangue ou noutro órgão extracutâneo. } \\
\text { d. Doseamento da triptase sérica }>20 \mathrm{ng} / \mathrm{mL} \text {, na ausência de neoplasia mieloide. }\end{array}$ \\
\hline
\end{tabular}

sistémica, é imprescindível a abordagem contínua e multidisciplinar dos doentes.

Este trabalho descritivo teve como objetivo a avaliação das características demográficas e clínicas de crianças com o diagnóstico de mastocitose.

\section{MATERIAL E MÉTODOS}

Foram revistos os processos clínicos, que remontam ao período de tempo compreendido entre janeiro de 2014 e junho de 2019, de todos os doentes com diagnóstico de mastocitose do Hospital Pediátrico de Coimbra, tendo sido analisados retrospetivamente os 14 doentes registados. Foi colhida informação, que incluiu: dados demográficos (sexo e idade), idade de início da apresentação clínica da mastocitose e idade ao diagnóstico, tipo de mastocitose, atingimento cutâneo e outra sintomatologia, agentes desencadeantes (calor, infeções, anestésicos gerais, fármacos, alimentos, picada de himenópteros), abordagem diagnóstica, abordagem terapêutica e evolução da doença. A classificação do tipo de mastocitose foi feita com base nos critérios estabelecidos pela OMS de $2016^{2}$.

\section{RESULTADOS}

Dos 14 doentes analisados, todos tinham mastocitose cutânea (MC), 12 (86\%) doentes na forma de MC maculopapular e 2 (14\%) na forma de mastocitoma cutâneo, $8(57 \%)$ do sexo masculino. Quanto à idade de início das lesões, a média foi de I5,I meses e a mediana de 5,0 meses ( $1 .^{\circ}$ dia de vida - 96 meses). A idade média de diagnóstico foi de 22,5 meses e a mediana de 9,5 meses (2-132 meses). A média do tempo decorrido entre o início das lesões e o diagnóstico foi de 7,4 meses. No que diz respeito à subclassificação das variantes da MC maculopapular, II foram classificadas como polimórficas e I como monomórfica. Em relação à localização das lesões houve um atingimento distinto do tronco, membros, cabeça, pescoço e face.

No que diz respeito à maioria das lesões cutâneas, estas variaram de máculas, pápulas e placas, com tonalidade variável (eritematosa a acastanhada), tamanho variável (infracentimétricas a I-2 centímetros). Nos doentes que apresentavam mastocitoma cutâneo, a lesão estava descrita como placa eritemato-acastanhada tipo couro e de forma ovalada, sendo que um dos doentes 
apresentava duas lesões. A par das lesões cutâneas foram descritos sinais e sintomas associados, nomeadamente o sinal de Darier, noção de prurido localizado às lesões por parte dos pais, dor abdominal e diarreia recorrente. No que concerne aos sintomas gastrointestinais associados, estes surgiam temporalmente associados ao trigger Em nenhum dos 14 doentes estava descrito episódio de anafilaxia. Foram reportados como trigger (desencadeante de desgranulação) o calor em 5 doentes, a ingestão alimentar (morango) em I doente e a patologia infeciosa em 4 doentes, nesta última mais concretamente a nasofaringite aguda. Durante o período a que remonta o presente estudo, no seguimento destes doentes não foi reportado agravamento da doença/intolerância à administração de fármacos, nomeadamente anti-inflamatórios não esteroides (AINES). Do mesmo modo, não havia registo em nenhum doente de fatores desencadeantes, como picada de himenópteros, administração de anestésicos gerais e/ou esforço físico. Em 2 doentes havia

Quadro 3. Análise das características dos 14 doentes estudados

\begin{tabular}{|c|c|}
\hline I. Idade de início das lesões & $\mathbf{N}(\%)$ \\
\hline $\begin{array}{l}\text { - Nascimento } \\
\cdot<6 \text { meses } \\
\cdot 6 \text { meses - I ano } \\
\cdot>\text { I ano }\end{array}$ & $\begin{array}{lr}1 & (7) \\
7 & (50) \\
3 & (22) \\
3 & (22)\end{array}$ \\
\hline 2. Idade de diagnóstico & $\mathbf{N}(\%)$ \\
\hline $\begin{array}{l}\cdot \leq \mathrm{I} \text { ano } \\
\cdot>\mathrm{I} \text { ano }\end{array}$ & $\begin{array}{r}10(71) \\
4(29)\end{array}$ \\
\hline 3. Localização das lesões & $\mathbf{N}(\%)$ \\
\hline $\begin{array}{l}\text { - Tronco } \\
\text { - Membros } \\
\text { - Cabeça/pescoço } \\
\text { - Face } \\
\cdot \geq 2 \text { localizações distintas }\end{array}$ & $\begin{array}{r}12(86) \\
10(71) \\
9(64) \\
2(14) \\
9(64)\end{array}$ \\
\hline 4. Sinais e sintomas associados & $\mathbf{N}(\%)$ \\
\hline $\begin{array}{l}\text { - Sinal de Darier } \\
\text { - Prurido } \\
\text { - Dor abdominal } \\
\text { - Diarreia } \\
\text { - Anafilaxia }\end{array}$ & $\begin{array}{l}12(86) \\
10(7 \mid) \\
2(14) \\
3(22) \\
-\end{array}$ \\
\hline
\end{tabular}

história familiar positiva de mastocitose cutânea, sendo desconhecida nos restantes. $\mathrm{O}$ Quadro 3 resume as características analisadas.

A abordagem diagnóstica contemplou um estudo inicial com hemograma, função renal, função hepática e ecografia abdominal, sendo que os resultados não foram sugestivos de envolvimento sistémico da doença. A triptase sérica foi doseada em todos os doentes, tendo oscilado entre 1,3-15,9 ng/mL na primeira avaliação, com apenas I caso de triptase sérica > II,4 ng/mL, sendo que o valor da triptase sérica é considerado normal quando $\leq \mathrm{II}, 4 \mathrm{ng} / \mathrm{mL}$. Neste caso em particular tratava-se de uma criança cujas lesões tiveram início ao nascimento, lesões descritas como múltiplas máculas e pápulas eritemato-acastanhadas dispersas por todo o tegumento, sem presença de outra sintomatologia, tendo-se verificado uma normalização do valor de triptase nos doseamentos subsequentes, sendo o último de $5,4 \mathrm{ng} / \mathrm{mL}$. Em 8 doentes, o diagnóstico foi confirmado através de biópsia cutânea. Nos doentes em que não se procedeu à biópsia cutânea, o diagnóstico foi assumido pelas características típicas das lesões e clínica dos doentes, sendo que 2 deles tinham mastocitoma cutâneo. Não foi requerida biópsia da medula óssea ou de outros órgãos extracutâneos, uma vez que os achados clínicos, analíticos e imagiológicos não eram sugestivos de envolvimento sistémico.

Fizeram parte da terapêutica instituída os corticosteroides tópicos de potência moderada a forte em SOS e os anti-histamínicos $\mathrm{H}_{1}$ per os, como o dimetindeno e a desloratadina, sendo que em 3 doentes foram administrados de forma diária para controlo dos sintomas e nos restantes apenas em SOS. Nos doentes com anti-histamínicos $\mathrm{H}_{1}$ diários a dose foi superior à estabelecida para a idade segundo o estipulado pela Autoridade Nacional do Medicamento e Produtos de Saúde, IP. A resposta à terapêutica conjuntamente com evicção do trigger foi favorável em todos os doentes do presente estudo, quer no controlo dos sintomas cutâneos, quer dos sintomas gastrointestinais. 
O follow-up era realizado entre 3 a 6 meses, de acordo com a evolução da doença, fazendo parte desse follow-up o doseamento da triptase sérica. Nos doentes em que a sintomatologia não estava controlada com anti-histamínico diário, houve a necessidade de reavaliar antes dos 6 meses. $O$ curso das lesões permanece estável em 12 doentes, sendo que nos outros 2 permanece por esclarecer o grau de estabilidade da doença por se tratarem de diagnósticos recentes. Nenhum doente entrou em remissão completa.

\section{DISCUSSÃo}

A mastocitose representa um grupo de doenças raras que podem ocorrer em qualquer idade, e que resultam da expansão de mastócitos clonais e da sua acumulação na pele (MC) e/ou em vários órgãos e sistemas ${ }^{5}$. Praticamente todas as crianças e mais de $80 \%$ dos adultos com mastocitose apresentam lesões cutâneas ${ }^{6}$. Nas crianças, o envolvimento cutâneo não é um fator preditivo para a existência de envolvimento sistémico, enquanto nos adultos está quase invariavelmente associado a envolvimento sistémico $^{10}$.

Este estudo descritivo permitiu analisar as características demográficas e clínicas da mastocitose em idade pediátrica. Como limitações deste trabalho mencionamos o facto de ser um estudo de natureza retrospetiva, o que não permite a elaboração do estudo de uma forma longitudinal e objetiva, e que limita o detalhe da obtenção dos dados relativos a aspetos práticos relevantes, como tolerância a fármacos, a alimentos, a picada a himenópteros, entre outros. Sendo estes fatores de objeto principal de avaliação do imunoalergologista e do seguimento clínico diferenciado requerido por estes doentes. Incluem-se também como limitações do estudo o número reduzido da amostra e altamente selecionada, contudo espectável dado tratar-se de uma doença pouco prevalente.

À semelhança do descrito na literatura, verificou-se que o total dos 14 casos corresponderam ao subtipo MC, sen- do a apresentação clínica mais frequente da mastocitose, com dois terços das MC a ocorrer em idade pediátrica tendo um bom prognóstico, com tendência à regressão espontânea durante a adolescência"'. Os estudos são discrepantes no que diz respeito à prevalência da mastocitose em idade pediátrica de acordo com o sexo, sendo que nos estudos mais recentes verifica-se uma predominância da mastocitose no sexo masculino até à adolescência, com uma reversão dessa tendência após a puberdade ${ }^{12}$. Neste estudo verificou-se uma ligeira predominância do sexo masculino com um rácio feminino/masculino de I/I,3.

A MC ocorre frequentemente na primeira infância, sendo frequente o início das lesões nos primeiros 6 meses de vida, incluindo aproximadamente $15 \%$ dos casos em que as lesões podem estar presentes ao nascimento ${ }^{6,9}$. Da mesma forma, verificamos que num doente (7\%) as lesões eram congénitas, $50 \%$ dos doentes tiveram o aparecimento das lesões antes dos 6 meses de idade, sendo que a maioria (78\%) teve a apresentação das lesões antes dos 12 meses. Em relação à idade do diagnóstico, são poucos os estudos que reportam dados sobre a relação temporal que decorre entre a idade de início das lesões e a idade em que é feito o diagnóstico. No presente trabalho, aproximadamente $71 \%$ dos doentes teve o diagnóstico feito antes do ano de idade, o que correspondeu ao número de doentes que iniciou o aparecimento de lesões no primeiro ano de vida. Por conseguinte, verificamos que, em média, o diagnóstico foi feito 7 meses depois do início das lesões.

De acordo com a classificação da OMS, as três principais formas de envolvimento cutâneo são a MC maculopapular, a MC difusa e o mastocitoma cutâneo, sendo a MC maculopapular a forma mais prevalente de mastocitose em idade pediátrica. No estudo de Gülen Tüsysüz et al., com uma amostra de 21 crianças, 90\% tinham MC maculopapular e os restantes $10 \%$ dos casos tinham mastocitoma cutâneo ${ }^{13}$. Noutro estudo semelhante de Hannaford et al., 5I\% dos doentes tinham mastocitoma cutâneo, 47\% MC maculopapular e apenas 2\% MC difusa ${ }^{14}$. No presente estudo, aproximadamente $85 \%$ dos casos 
tinham MC maculopapular e 14\% mastocitoma cutâneo, não existindo nenhum caso com mastocitose difusa. À semelhança do que está descrito na literatura em que, na criança, a distribuição das lesões na variante polimórfica da MC maculopapular atinge habitualmente tronco, membros, cabeça e pescoço, ${ }^{6,15}$, também neste estudo verificamos que as localizações mais frequentemente atingidas foram o tronco e os membros.

O sinal de Darier, isto é, o desenvolvimento de eritema, edema e/ou urticária 5 a 15 minutos após fricção sobre a lesão cutânea, é um achado patognomónico da libertação de mediadores dos mastócitos cutâneos ${ }^{12,16}$. Este sinal é frequentemente positivo na população pediátrica com o diagnóstico de $\mathrm{MC}^{17}$ e, quando pesquisado em lesões de grandes dimensões e em doentes particularmente sensíveis, pode desencadear anafilaxia ${ }^{10}$. De forma concordante com o que está descrito na literatura, o sinal de Darier estava presente em 12 doentes. Estão também descritos outros sinais e sintomas associados, que podem incluir prurido, sintomatologia gastrointestinal e, raramente, dificuldade respiratória, mal-estar geral, fadiga e cefaleia ${ }^{5}$. No grupo de doentes estudado foi predominante a presença de prurido (7I\%) localizado às lesões e sintomatologia gastrointestinal (36\%), nomeadamente dor abdominal e diarreia. Não foi reportado nenhum episódio de anafilaxia, entidade esta raramente observada na mastocitose em idade pediátrica. São vários os triggers associados à ativação/libertação de mastócitos, como os estímulos mecânicos, o calor, o exercício físico, os fármacos, a picada de himenópteros e as infeções ${ }^{18}$. Nesta amostra, os triggers reportados foram calor, sobretudo associado à temperatura quente do banho, ingestão alimentar e infeções associadas ao trato respiratório superior. Não foi reportado agravamento da doença/intolerância à administração de fármacos, nomeadamente anti-inflamatórios não esteroides, não havia registo em nenhum doente de fatores desencadeantes, como picada de himenópteros, administração de anestésicos gerais e/ou esforço físico. A mastocitose normalmente ocorre de forma esporádica e raramente de forma hereditária ${ }^{19}$. Em relação à história familiar, a presença de atingimento cutâneo similar nos familiares foi descrita em dois casos.

Apesar de ser menos comum a presença de MS em idade pediátrica, foi feita uma avaliação inicial em todos os doentes estudados, com hemograma, função renal, função hepática, doseamento de triptase sérica e ecografia abdominal, de forma a excluir o atingimento sistémico da doença, não tendo sido reportadas alterações sugestivas desse atingimento. $O$ doseamento da triptase sérica reflete a carga total de mastócitos, sendo que a sua determinação parece ser uma ferramenta útil ao diagnóstico e monitorização dos doentes com MC, dada a sua estreita relação com o curso da doença e a sua gravidade ${ }^{12}$. Consideram-se como níveis normais de triptase sérica os valores $\leq \mathrm{II}, 4 \mathrm{ng} / \mathrm{mL}$, níveis estes que, segundo a literatura, são os que se verificam na MC; no entanto, níveis de triptase baixos não excluem o envolvimento sistémico ${ }^{20}$. Por outro lado, níveis superiores a $>20 \mathrm{ng} / \mathrm{mL}$ são sugestivos do diagnóstico de MS; contudo, níveis de triptase $>20 \mathrm{ng} / \mathrm{mL}$ podem ser observados em doentes com MC e atingimento cutâneo extenso ${ }^{6,21}$. Consideram-se como achados clínicos característicos do envolvimento sistémico a presença de sintomas gastrointestinais exuberantes, história de episódios de anafilaxia, episódios de anafilaxia associados à picada de himenópteros, anestésicos gerais e outros fármacos, palpação de adenomegalias ao exame objetivo, hepatomegalia e/ou esplenomegalia, também achados analíticos, como citopenias ou alterações na bioquímica hepática, achados imagiológicos, como hepatomegalia e/ou esplenomegalia ${ }^{12,22,23}$. Tendo em consideração a baixa incidência de MS em idade pediátrica, na maioria dos casos só é feito o estudo analítico e doseamento dos níveis de triptase sérica, sendo a biópsia da medula óssea realizada apenas em casos selecionados, ou seja, aqueles em que há suspeita de envolvimento sistémico. Na maioria dos casos do presente estudo, o doseamento da triptase sérica foi normal na primeira avaliação e a biópsia cutânea, para estabelecimento do diagnóstico definitivo, foi efetuada em 8 doentes, não tendo sido realizada biópsia da medula óssea em nenhum dos doentes. 
O foco do tratamento contempla a evicção dos fatores desencadeantes, o alívio sintomático e prevenção da libertação de mediadores mastocitários. Fazem parte da terapêutica farmacológica, na maioria dos casos, os anti-histamínicos $\mathrm{H}_{1}$, os anti-histamínicos $\mathrm{H}_{2}$, os corticosteroides tópicos e o cromoglicato de sódio ${ }^{16,10}$. Os inibidores da calcineurina tópicos têm tido um papel crescente e seguro no tratamento da MC localizada, como alternativa aos corticosteroides tópicos ${ }^{24}$.

Ainda que na maioria dos casos em idade pediátrica a mastocitose seja cutânea, é imprescindível no follow-up destes doentes a avaliação e monitorização dos fatores desencadeantes dos episódios, a avaliação da regressão/estabilidade das lesões e o doseamento da triptase sérica.

\section{CONCLUSÕES}

Como descrito na literatura, o presente trabalho reforça que a mastocitose em idade pediátrica é claramente dominada pela MC. Urge a necessidade de uma maior definição e categorização da $M C$, bem como de perceber a incidência precisa desta doença em idade pediátrica e do tratamento correto. Este trabalho realça a importância do conhecimento das características da doença em idade pediátrica para o diagnóstico, investigação e tratamento dirigidos, assim como a sua abordagem multidisciplinar, de forma atingir a estabilidade clínica destes doentes.

\section{Conflito de interesses}

Os autores declaram que não existem conflitos de interesses.

\section{Contacto:}

Francisca da Cunha Tavares

Serviço de Imunoalergologia,

Centro Hospitalar e Universitário de Coimbra

Praceta Prof. Mota Pinto 3000-075 Coimbra

Francisca.cunha.tavares@gmail.com

\section{REFERÊNCIAS}

I. Crivellato E, Beltrami C, Mallardi F, Ribatti D. Paul Ehrlich's doctoral thesis: a milestone in the study of mast cells. $\mathrm{Br} \mathrm{J}$ Haematol. 2003 Oct;123(I):19-2I. doi: 10.1046/j.I365-2|4I.2003.04573.x

2. Valent P, Akin C, Metcalfe DD. Mastocytosis: 2016 updated WHO classification and novel emerging treatment concepts. Blood 2017;129:1420-7.

3. Theoharides TC, Valent P, Akin C. Mast cells, mastocytosis, and related disorders. N Engl J Med 2015;373:163-72.

4. Matito A, Azaña JM, Torrelo A, Alvarez-Twose I. Cutaneous mastocytosis in adults and children: New classification and prognostic factors. Immunol Allergy Clin North Am 2018;38:35163.

5. Abid A, Malone MA, Curci K. Mastocytosis. Prim Care-Clin Off Pract 2016;43:505-18.

6. Ferreira S, Fernandes I, Cabral R, Machado S, Lima M, Selores M. Cutaneous manifestations in mastocytosis: Update. Acta Med Port 2020;33:275-81.

7. Pardanani A. Systemic mastocytosis in adults: 2019 update on diagnosis, risk stratification and management. Am J Hematol 2019;94:363-77.

8. Cohen SS, Skovbo S, Vestergaard H, Kristensen T, Moller M, Bindslev-Jensen C, et al. Epidemiology of systemic mastocytosis in Denmark. Br J Haematol 2014;166:52I-8.

9. Broesby-Olsen S, Medoly C, Kjaer HF, Mortz CG, Moller MB, Kristensen TK, et al. Pediatric expression of mast cell activation disorders. Immunol Allergy Clin North Am 2018;38:365-77.

10. Rama T, Moreira A, Delgado L. Abordagem diagnóstica e terapêutica das mastocitoses - Uma proposta de orientação clínica. Rev Port Imunoalergologia 2020;28:31-49.

II. Lange M, Niedoszytko B, Górska A, Zawrocki A, Sobjanek M, Kozłowski D. Mastocytosis in children and adults: Clinical disease heterogeneity. Arch Med Sci 2012;8:533-4I.

12. Klaiber N, Kumar S, Irani AM. Mastocytosis in children. Curr Allergy Asthma Rep 2017;17:1-8.

13. Tüysüz G, Özdemir N, Apak H, Kutlubay Z, Dermirkesen C, Celkan T. Childhood mastocytosis: Results of a single center. Turk Pediatr Ars 2015;50:108-13.

14. Hannaford R, Rogers M. Presentation of cutaneous mastocytosis in 173 children. Australas J Dermato 2001;42:15-21.

I5. Hartmann K, Escribano L, Grattan C, Brockow K, Carter M, Alvarez-Twose I, et al. Task force report Cutaneous manifestations in patients with mastocytosis: Consensus report of the European Competence Network on Mastocytosis. American Academy of Allergy, Asthma \& Immunology and the European Academy of Allergology and Clinical Immunology. J Allergy Clin Immunol 20I6;137(I):35-45. doi: 10.1016/j.jaci.2015.08.0342008;|-II

16. Castells M, Metcalfe DD, Escribano L. Guidelines for the diagnosis and treatment of cutaneous mastocytosis in children. Am J Clin Dermatol 20II;12:259-70. 
17. Lange M, Lugowska-Umer H, Niedoszytko M, Wasag B, Limon J, Zawrocki A, et al. Diagnosis of mastocytosis in children and adults in daily clinical practice. Acta Derm Venereol 2016;6:2927.

18. Gümüșburun R, Mușabak UH, Bavbek S. Mastocytosis. Arch Argent Pediatr 2019;17:172-3.

19. Longley BJ, Metcalfe DD, Tharp M, Wang X, Tyrrell L, Lu S-H, et al.Activating and dominant inactivating c- KIT catalytic domain mutations in distinct clinical forms of human mastocytosis. Proc Natl Acad Sci USA 1999;96:1609-14.

20. Alvarez-Twose I, Jara-Acevedo M, Morgado JM, García-Montero A, Sánchez-Muñoz L, Teodósio C, et al. Clinical, immunophenotypic, and molecular characteristics of well-differentiated systemic mastocytosis. J Allergy Clin Immunology 2016;137(I):168-78.el.
21. Frieri M, Quershi M. Pediatr Allergy Immunol Pulmonol 2013;26(4):175-80. doi: 10.1089/ped.2013.0275

22. Potier A, Lavigne C, Chappard D, Verret JL, Chevailler CA, Nicolie $\mathrm{B}$, et al. Cutaneous manifestations in hymenoptera and diptera anaphylaxis: relationship with basal serum tryptase. Clin Exp Allergy 2009;39:717-25.

23. Hartmann K, Metcalfe DD. Pediatric mastocytosis. Hematol Oncol Clin North Am 2000;14(3):625-40. doi: 10.1016/s0889-8588(05)70299-9

24. Correia O, Duarte AF, Quirino P, Azevedo R, Delgado L. Cutaneous mastocytosis: Two pediatric cases treated with topical pimecrolimus. Dermatol Online J 2010;16(5):8. PMID: 20492825 